E-mail: conny.turni@dpi.qld.gov.au

\title{
An evaluation of the apxIVA based PCR-REA method for differentiation of Actinobacillus pleuropneumoniae
}

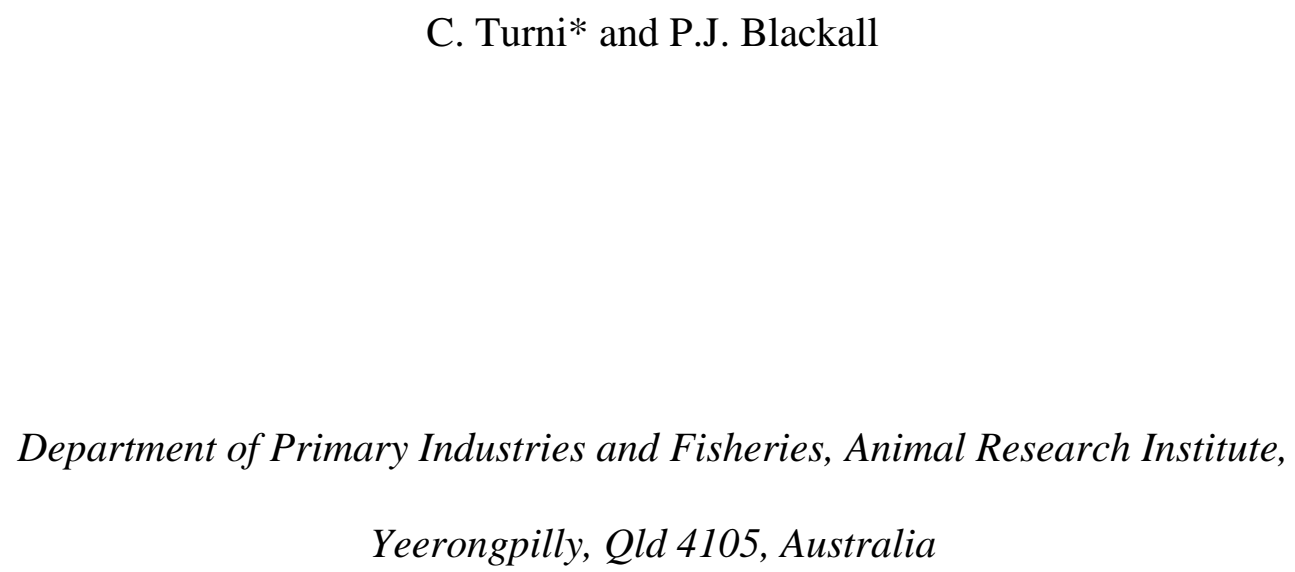


1 Abstract

2

3

4

5

A restriction analysis of PCR (PCR-REA) amplified apxIVA gene has been suggested as an alternative method for serotyping of Actinobacillus pleuropneumoniae by Jaglic et al. (2004). The current study investigated whether this alternative method could distinguish between the reference strains of serovar 13, 14 and 15 and the value of the method when applied to 47 field isolates representing serovars 1, 2, 3, 5, 7, 8, 9, 12 and 15 as well as non-typable isolates. The reference strains of serovars 13 and 14 had the same sized product after the apxIVA PCR, while the product for serovar 15 was of different size compared to all the other serovar reference strains. The CfoI digest profiles of the reference serovar 13 and 14 strains were different from each other and from all other serovars. The HpaII digest profiles of these two serovars were very similar to each other, but both were distinctively different from the other serovar profiles. The $C f o I$ digest profile of serovar 15 strain was very similar to the serovars 3 and 12 strains except for two faint extra bands for serovar 15. The HpaII digest profiles of serovar 12 and 15 reference strains were identical. The PCR-REA method correctly recognized the serovar of 21 of 43 field isolates. It was concluded that the method was a useful additional tool to support, but could not replace, conventional serotyping.

Keywords: Actinobacillus pleuropneumoniae; apxIVA; PCR-REA; CfoI; HpaII 


\section{Introduction}

Actinobacillus pleuropneumoniae, the causative agent of porcine pleuropneumonia, contributes substantially to economic losses in the swine industry worldwide (Nicolet, 1992). Nielsen et al. (1997) proposed the integration of the serotyping scheme for A. pleuropneumoniae, which was previously split into two biovars 1 and 2, which resulted in the recognition of serovars 1 to 14 . Serovar 15 was described by Blackall et al. (2002).

Serotyping for Actinobacillus pleuropneumoniae is complicated by crossreactions amongst strains of serovars (Dubreuil et al., 2000). Strong cross-reactions are observed between serovars 1, 9 and 11; 3, 6 and 8 and 4 and 7 (Mittal et al., 1988; Mittal and Bourdon, 1991; Mittal et al., 1993). In a recent study by Kucerova et al. (2005), 245 isolates of A. pleuropneumoniae were serotyped with $7.8 \%$ displaying a two-way cross-reaction between serovar 9 and 11 . The history of serovar 15 is another example of the difficulties with serotyping cross-reactions. The isolates obtained from Australian pigs that are now recognized as serovar 15 were originally assigned to serovar 12 (Blackall et al., 2002).

Jaglic et al. (2004) proposed a restriction enzyme analysis of PCR amplified apxIVA gene (PCR-REA) as an alternative method to serotyping for differentiating serovars of A. pleuropneumoniae. In their study, Jaglic et al. (2004) examined the reference strains of serovar 1 to 12 as well as field isolates that were cross-reactive with antisera to serovars 1, 9 and 11. In the current study, the work of Jaglic et al. (2004) is extended by examining the reference strains of serovars 13 to 15 and analyzing 40 field isolates (of various serovars) from Australia, 6 from Indonesia and 
one from New Zealand to investigate whether the method could be used as an alternative to conventional serotyping of A. pleuropneumoniae in Australia.

\section{Materials and Methods}

\subsection{Bacterial strains, media and growth conditions}

The reference strains of A. pleuropneumoniae, held in the Animal Research Institute culture collection, were (in order of 1 to 15) - 4074, 4226, 1421, M 62, K17, Femo, WF 83, 405, CVJ 13261, 13039, 56153, 1096, N 273, 3906 and HS 143. A total of 47 pig field isolates of A. pleuropneumoniae were examined (40 from Australian, six from Indonesia and one from New Zealand). All the field isolates were confirmed as A. pleuropneumoniae by phenotypic methods as previously described (Blackall and Pahoff, 1995) or by the PCR described by Gram and Aherns (1998). All the field isolates were serotyped by either the gel diffusion and/or the indirect haemagglutination tests using antisera to the reference strains, listed above, for serovars 1 to 12 and 15 as previously described (Eaves and Blackall, 1988; Blackall and Pahoff, 1995; Blackall et al., 2002). Full details of the field isolates are in Table 1.

All bacteria were grown at $37^{0} \mathrm{C}$ on $\mathrm{BA} / \mathrm{SN}$ agar, which consists of $\mathrm{BBL}^{\mathrm{TM}}$ Blood Agar Base (Becton Dickinson, Sparks MD USA). Just before pouring the media was supplemented with $0.0025 \%$ of $\mathrm{NADH}, 0.0005 \%$ of Thiamine $\mathrm{HCl}, 1 \%$ of heat inactivated horse serum and $5 \%$ of oleic acid bovine albumin complex which consists of $4.75 \%$ bovine serum albumin (fraction $\mathrm{V}$ ) in normal saline (with the normal saline containing $0.06 \%$ oleic acid and $5 \% 0.05 \mathrm{~N} \mathrm{NaOH}$ ). 
A $1 \mu \mathrm{l}$ loopful of A. pleuropneumoniae from overnight culture was thoroughly suspended in $100 \mu \mathrm{l}$ ultrapure water, vortexed and kept on ice for $5 \mathrm{~min}$. The suspension was heated at $98^{\circ} \mathrm{C}$ for $5 \mathrm{~min}$, followed by cooling on ice for $5 \mathrm{~min}$. After another 5 min at $98^{\circ} \mathrm{C}$ the solution was centrifuged ( 2 min at $30230 \mathrm{x}$ ) ) and the supernatant collected and stored at $-20^{\circ} \mathrm{C}$. This method was slightly altered for both reference and field samples of serovar 7 and 15 , where cultures were heated at $98^{\circ} \mathrm{C}$ for $1 \mathrm{~min}$ instead of $5 \mathrm{~min}$.

A $2 \mu \mathrm{l}$ aliquot of the supernatant was used for PCR analysis. The PCR was performed as previously described by Jaglic et al. (2004) with the following alterations. The HotStar Taq Master Mix Kit (QIAGEN, Hilden, Germany) was used instead of the Taq PCR Master Mix Kit. The cycling parameters of the PCR were changed to $95^{\circ} \mathrm{C}$ for $15 \mathrm{~min}$, followed by 35 cycles at $94^{\circ} \mathrm{C}$ for $45 \mathrm{sec}, 59^{\circ} \mathrm{C}$ for 45 sec and $72^{\circ} \mathrm{C}$ for $6 \mathrm{~min}$. The final elongation step was at $72^{\circ} \mathrm{C}$ for $10 \mathrm{~min}$. Amplification products were visualised by electrophoresis as described (Jaglic et al. 2004).

The digestion of $20 \mu \mathrm{l}$ PCR product with $6 \mathrm{U}$ of $C$ foI or $10 \mathrm{U}$ of HpaII (Roche, Mannheim, Germany) was done for 2 hrs at $37^{\circ} \mathrm{C}$. Visualisation was on a $1.5 \%$ agarose gel run for $1.5 \mathrm{hrs}$ at $80 \mathrm{~V}$. 
The molecular weight of the apxIVA PCR and the bands generated by the subsequent restriction digests were analysed with BioNumerics software (Applied Maths Inc, Sint-Martens-Latem, Belgium).

\section{Results}

The reference strains and field isolates of serovar 7 and 15 gave very poor yields of PCR product when the cells were heated at $98^{\circ} \mathrm{C}$ for $5 \mathrm{~min}$. When the incubation time was reduced to 1 min, much better yields of PCR product were obtained.

Running the apxIVA PCR product of the 15 reference strains on a gel produced seven different bands. The reference strains of serovar 2, 5, 7 and 8 produced PCR products of the same molecular size of 3,929-3,978 kb. PCR of serovars 1, 3, 12, 13 and 14 gave a PCR product of 3,551 - 3,599 kb. Serovars 6 and 10 yielded PCR products of the same band size of 3,066 and 3,044 kb respectively. Serovars 4, 9, 11 and 15 had unique PCR products (2,877, 2,711, 2,535 and 4,390 kb respectively) that differed from all the other serovar PCR products.

The results of the $C f o$ I digest of the apxIVA PCR products of the 15 reference strains are shown in Table 2, displayed according to the band matching results of the BioNumerics program. According to detailed profile analysis all 15 reference strains could be distinguished from each other. However, the simple visual examination of the gel picture highlighted that three pairs of serovars would be difficult to distinguish. These difficult pairs were serovars 5 and 7, 8 and 10 and 9 and 11 . The gel profile for serovars 3 and 12 was identical and only the low molecular bands of 68 
1 and 74 bp hold them apart in the BioNumerics profile (Table 2). The profiles for serovars 13 and 14 were distinct from the profiles for the other serovars (Table 2). The profile of serovar 15 was very similar to that of serovars 3 and 12, except that serovar 15 had two faint bands of approximately $400 \mathrm{bp}$.

The results of the HpaII digest of the apxIVA PCR product of the 15 reference strains are shown in Table 3 according to the band matching results of the BioNumerics program. The profile for serovar 5 was very similar to the profile for serovar 7 except for one faint band at 225 bp for serovar 7 . The profiles of serovars 8 and 10 were clearly distinguishable from each other. The profiles of serovar 9 and 11 were not clearly distinguishable, especially on visual examination. The profiles of serovars 12 and 15 were nearly identical but could be distinguished from serovar 3. The HpaII profiles for serovars 13 and 14 were very similar, but both were distinctively different from the other serovar profiles. However, if the faint bands around the 2,000 and 1,500 bp were not included, then the profile would be very similar to that of serovar 5 and 10, similar to serovar 6 and similar to serovars 2 and 7 , both of which have one extra faint band (Table 3).

The results for the field isolates were not as conclusive as the reference strains (Table 1). A summary is shown in Table 4. Overall, of the 43 isolates that were confidently assigned to a serovar by the traditional serotyping tests, 21 were assigned to the same serovar by the PCR-REA method. A further seven isolates were nearly matched using the PCR-REA method. A near match was defined as meaning that the PCR-REA method suggested more than one serovar with one of those serovars being the same as that given by the traditional methods. 
The PCR-REA method performed poorly with serovar 1 - none of the 8

isolates were correctly assigned and only two were a near match. A similar poor association was found with serovar 3 - three isolates were mismatched and one isolate was a near match. In contrast, the PCR REA method performed well with serovars 8 (all seven isolates correctly assigned) and 15 (seven of eight isolates correctly assigned). Of the other serovars represented by four or more isolates, PCR-REA gave near matches with most serovar 12 isolates (three of four) and correct or near matches with serovars 5 and 7.

\section{Discussion}

Several differences between the study by Jaglic et al. (2004) and the current study were observed. Jaglic et al. (2004) only reported two sizes of the amplicon, 3,000 and 3,529 bp for serovars 1 to 12, while the current study observed 6 different bands for these serovars. Amplicon size differences were between 289 to 465 bp for the reference strains of serovars 2, 5, 7, 8, 9, 10 and 11, despite both this study and Jaglic et al. (2004) using the same reference strains.

The $C f o$ I digest profile for reference serovar 5 was similar to serovar 7 in the current study. Three of the field isolates of serovar 5 gave the same profile as the reference serovar 5 strain, while the other three field serovar 5 isolates gave the profile of reference serovar 6 strain. In contrast Jaglic et al. (2004) reported the profile of serovar 6 strain was similar to that of serovar 7 with the exception of an extra band ( 1,500 bp) for serovar 6. Unfortunately, Jaglic et al. (2004) did not use the PCR-REA method on any field isolates of serovars 5 or 6 . 
Another difference between the studies was the presence of two bands around

In the current study, the $C f o$ I profile did not allow for a visual separation of the reference strains for serovars 3 and 12. Jaglic et al. (2004) made no note of this difficulty and simply reported that the two strains were distinct.

This study encountered the same difficulty in differentiating between serovars 9 and 11 as reported by Jaglic et al. (2004). However, the difference in the PCR amplicon size found in this study did allow the separation of the two serovars. The serological cross-reactions between serovars 1, 9 and 11 and the genetic relationship between serovars 9 and 11 are well recognised (Chevallier et al., 1998; Dubreuil et al., 2000). The serological cross-reactions between serovars 1,9 , and 11 are a reflection of similarities in the lipopolysaccharide O-antigen (Jacques, 2004; Perry et al., 2005). Therefore, the major difficulties existing in recognizing serovar 1 field isolates by the PCR-REA method, which assigned most of the isolates to serovar 9 or 11, are not surprising.

The finding of similarities in the REA profiles of the reference strains for serovars 5 and 7 and for 8 and 10 are not seen in serotyping methodologies, whereas serovar 7 cross-reacts with serovar 4 (Perry et al., 2005) and serovar 8 cross-reacts 
1 with serovar 6 (Perry et al., 2005). However, Musser et al. (1987), on the basis of a

6 multi-locus enzyme electrophoresis study, found a close relationship between isolates within serovars 1, 5 and 7 and Chevallier et al. (1998) found a relationship between serovars 8 and 10 by PFGE.

Two of the serovar 5 field isolates were assigned to serovar 6 in the PCR-REA method. Chevallier et al. (1998), using PFGE, found a relationship between serovars 5 and 6.

There is now considerable evidence that the population structure of $A$. pleuropneumoniae is clonal (Musser et al. 1987; Hampson et al. 1993; Chevallier et al. 1998) and that unrelated clones may be serologically identical (Chevallier et al., 1998). This clonal structure may explain while the PCR-REA method can correctly assign the serovar of some field isolates, yet fail for other isolates within the same serovar.

\section{Other studies looking at alternative methods, like outer membrane lipoprotein} (omlA) PCR typing system in combination with an apx (apx I, II, and III) typing system and apx PCR typing system including primers for all apx genes, coud not distinguish between serovars 1, 9 and 11 and 2 and 8 (Gram et al 2000).

In conclusion the PCR-REA method of Jaglic et al. (2004) cannot be used as a substitute for conventional serotyping of A. pleuropneumoniae. However, the method does have the potential to be an additional tool to support serotyping, particularly for 
serovars 2, 5, 7, 8, 12 and 15 . The method might be helpful to shed some light on clonal relationships between samples.

\section{Acknowledgment}

We would like to thank Matthew Pyke for his valuable technical work.

\section{References}

Blackall, P.J., Pahoff, J., 1995. Characterisation of porcine haemophili isolated from Australian pigs, 1988-1992. Aust. Vet. J. 72, 18-21.

Blackall, P.J., Klaasen, H.L.B.M., Van Den Bosch, H., Kuhnert, P., Frey, J., 2002. Proposal of a new serovar of Actinobacillus pleuropneumoniae: serovar 15. Vet. Microbiol. 84, 47 - 52.

Boekema, B.K.H.L., Van Putten, J.P.M., Stockhofe-Zurwieden, N., Smith, H.E., 2004. Host cell contact-induced transcription of the Type IV Fimbria gene cluster of Actinobacillus pleuropneumonia. Infect. Immun. 72, 691-700.

Chevallier, b., Dugourd, D., Tarasiuk, K., Harel, J., Gottshalk, M., Kobisch, M., Frey, J., 1998. Chromosome size and phylogenetic relationships between serotypes of Actinobacillus pleuropneumoniae. FEMS Microbiol. Letters 160, 209 216.

Dubreuil, D.J., Jacques, M., Mittal, K.R., Gottschalk, M., 2000. Actinobacillus pleuropneumoniae surface polysaccharides: their role in diagnosis and immunogenicity. Anim. Health Res. Rev., 1, 73 - 93.

Eaves, L.E., Blackall, P.J., 1988. Serological characterisation of Australian isolates of Actinobacillus pleuropneumoniae. Aust. Vet. J. 65, 379-381. 
1 Gram, T., Ahrens, P., 1998. Improved diagnostic PCR assay for Actinobacillus pleuropneumoniae based on the nucleotide sequence of an outer membrane lipoprotein. J. Clin. Microbiol. 36, 443-448.

Gram, T., Ahrens, P., Andreasen, M., Nielsen, J.P., 2000. An Actinobacillus pleuropneumoniae PCR typing system based on the apx and omlA genes evaluation of isolates from lungs and tonsils of pigs. Vet. Microbiol. 75, 43 57.

Hampson, D. J., Blackall, P. J., Woodward, J. M., Lymbery, A., J. 1993. Genetic analysis of Actinobacillus pleuropneumoniae, and comparison with Haemophilus spp. Taxon "minor group" and Taxon C. Zentralbl. Bakteriol. 279, 83-91.

Jacques, M., 2004. Surface polysaccharides and iron-uptake systems of Actinobacillus pleuropneumoniae. Can. J. Vet. Res. 68, 81 - 85.

Jaglic, Z. Svastova, P., Rychlik, I., Nedbalcova, K. Kucerova, Z, Pavlik, I., Bartos, M., 2004. Differentiation of Actinobacillus pleuropneumoniae by PCR-REA based on sequence variability of the apxIVA gene and by ribotyping. Vet. Microbiol. 103, 63 -69.

Kucerova, Z., Jaglic, R., Ondriasova, R., Nedbalcova, K., 2005. Serotype distribution of Actinobacillus pleuropneumoniae in Czech Republic during period 2003 2004. Vet. Med. - Czech 50, 355 - 360.

Mittal, K.R., Bourdon, S., 1991. Cross-reactivity and antigenic heterogeneity among Actinobacillus pleuropneumoniae strains of serotypes 4 and 7. J. Clin. Microbiol. 29, $1344-1347$. 
Mittal, K.R., Kamp, E.M., Kobisch, M., 1993. Serological characterization of Actinobacillus (Haemophilus) pleuropneumoniae strains of serotypes 1, 9 and 11. Res. Vet. Sci. 55, $179-184$.

Mittal, K.R., Higgins, R., Lariviere, S., 1988. Quantification of serotype and cross reacting group specific antigens by coagglutination and immunodiffusion tests for different Actinobacillus (Haemophilus) pleuropneumoniae strains belonging to cross reacting serotypes 3, 6 and 8. J. Clin. Microbiol. 26, 985 989.

Musser, J. M., Rapp, V. J., Selander, R.K., 1987. Clonal Diversity in Haemophilus pleuropneumoniae. Infect. Immun. 55, 1207 -1215.

Nicolet, J., 1992 Actinobacillus pleuropneumoniae. In: Leman, A.D., Straw, B.E., Mengeling, W.L., D’Allaire, S., Taylor, D.J. (Eds.), Diseases of Swine, Iowa State University Press, Ames, IO, pp. 401 - 408.

Nielson, R., Andresen, L.O., Plambeck, T., Nielsen, J.P., Krarup, L.T., Jorsal, S.E., 1997. Serological characterization of Actinobacillus pleuropneumoniae biotype 2 strains isolated from pigs in two Danish herds. Vet. Microbiol. 54, $35-46$.

Perry, M. B., MacLean, L.L., Vinogradov, E., 2005. Structural characterization of the antigenic capsular polysaccharide and lipopolysaccharide O-chain produced by Actinobacillus pleuropneumoniae serotype 15. Biochem. Cell Biol. 83, 61 $-69$. 
Table 1 Comparison of conventional serotyping (by GD or IHA) and serovar assignment by

2 PCR-REA of the apxIVA gene using either $C f o \mathrm{I}$ or $H p a I^{\mathrm{a}}$.

\begin{tabular}{|c|c|c|c|c|}
\hline \multirow[b]{2}{*}{ Field Isolates $^{\mathrm{a}}$} & \multirow{2}{*}{$\begin{array}{l}\text { GD /IHA } \\
\text { serovar }\end{array}$} & \multicolumn{3}{|c|}{ Serovar based on } \\
\hline & & $\begin{array}{c}\text { Size of apxIVA } \\
\text { product }\end{array}$ & CfoI digest & HpaII digest \\
\hline 25 & $1^{\mathrm{b}}$ & $1,3,12,13$ or 14 & 9 & 1 or 3 \\
\hline 1851 & 1 & $1,3,12,13$ or 14 & 11 & 4,9 or 11 \\
\hline 1893 & 1 & $1,3,12,13$ or 14 & 11 & 1 or 3 \\
\hline 2034 & 1 & 11 & 11 & 4,9 or 11 \\
\hline 2043 & 1 & 11 & 9 & 4,9 or 11 \\
\hline 2089, 2097, 2099 & 1 & 6 or 10 & 10 & 14 \\
\hline 588,984 & 2 & 2 & 2 & $\mathrm{ND}^{\mathrm{e}}$ \\
\hline 77 & $3^{\mathrm{b}}$ & $2,5,7$ or 8 & $?^{\mathrm{d}}$ & ND \\
\hline 612 & 3 & 15 & 15 & ND \\
\hline 721 & 3 & $1,3,12,13$ or 14 & 3 or 12 & 1 or 3 \\
\hline 523 & $3+6$ & ? & ? & ND \\
\hline 1373,2230 & 5 & $2,5,7$ or 8 & 5 & ND \\
\hline 1852,2018 & 5 & 6 or 10 & 6 & ND \\
\hline 2214 & 5 & $2,5,7$ or 8 & 5 & $?$ \\
\hline 30 & $7^{\mathrm{b}}$ & $2,5,7$ or 8 & 7 & ND \\
\hline 1861 & 7 & ? & 5 or 7 & $?$ \\
\hline 1891 & 7 & $2,5,7$ or 8 & 7 & ND \\
\hline $\begin{array}{l}1051 \\
2325\end{array}$ & 7 & ? & ? & ND \\
\hline 1172, 2054, 2074, 2075 & 8 & $2,5,7$ or 8 & 8 & 8 \\
\hline 1383 & 8 & $2,5,7$ or 8 & 8 & ND \\
\hline $\begin{array}{l}1303 \\
1926\end{array}$ & $\begin{array}{c}0 \\
6+8\end{array}$ & $2,5,7$ or 8 & 8 & ND \\
\hline 1965 & $8(6)$ & $2,5,7$ or 8 & 8 & ND \\
\hline $\begin{array}{c}1905 \\
17\end{array}$ & $\begin{array}{c}0(0) \\
9^{b}\end{array}$ & $2,5,7$ or 8 & ? & 2,5 or 10 \\
\hline 195, 199 & $12(15)$ & ? & 3 or 12 & 1 or 3 \\
\hline 872 & 12 & $1,3,12,13$ or 14 & 3 or 12 & 1 or 3 \\
\hline 1413 & 12 & 6 or 10 & $?$ & ? \\
\hline 160 & $15^{\mathrm{c}}$ & $?$ & 15 & ND \\
\hline 173,451 & 15 & $?$ & 15 & ND \\
\hline 1937 & 15 & 11 & 4 & 4,9 or 11 \\
\hline 2235, 2333, 2334, 2335 & 15 & 15 & 15 & ND \\
\hline 409 & NT & 15 & 15 & ND \\
\hline 2038 & NT & $1,3,12,13$ or 14 & 3 or 12 & ? \\
\hline 2192 & NT & ? & 7 & 7 \\
\hline 2207 & NT & ? & 4 & ND \\
\hline
\end{tabular}

${ }^{\mathrm{a}}$ All isolates were from Australian pigs except that isolates 2054, 2074, 2075, 2089, 2097 and 2099 which were from Indonesian pigs and isolate 2230 which was from a New Zealand pig

${ }^{\mathrm{b}}$ These isolates have been confirmed as the indicated serovar by a second laboratory (Boekema et al., 2004)

${ }^{\mathrm{c}}$ This isolate has been confirmed as serovar 15 by a second laboratory (Blackall et al. 2002)

${ }^{\mathrm{d}}$ ? = no match with any band or profile type seen in the 15 reference strains

${ }^{\mathrm{e}} \mathrm{ND}=$ not done 
Table 2 Molecular weight of bands following $C f o$ I digestion of the apxIVA amplification product of the reference strains of A. pleuropneumoniae.

\begin{tabular}{|c|c|c|c|c|c|c|c|c|c|c|c|c|c|c|}
\hline \multicolumn{15}{|c|}{ Size (kb) of bands following $C f o$ I digestion of apxIVA PCR product of the reference strain of the indicated A. pleuropneumoniae serovar } \\
\hline 1 & 2 & 3 & 4 & 5 & 6 & 7 & 8 & 9 & 10 & 11 & 12 & 13 & 14 & 15 \\
\hline & & & & & & & & & & & & & 2988 & 2504 \\
\hline & & 2375 & 2383 & & & & & & & & 2392 & & & \\
\hline & & & & & & & & $2194^{\mathrm{a}}$ & & $2275^{\circ}$ & & & & \\
\hline & & & & 1095 & & & & & & & & 1961 & & \\
\hline & 1812 & & & & & & & & & & & & & \\
\hline & & & & & $1 / 89$ & 1704 & 1750 & & 1723 & & & $1742^{\mathrm{a}}$ & & \\
\hline & & & & & & & & 1520 & & & & & & \\
\hline & & & & & & & & & & 1448 & & $1206^{a}$ & & \\
\hline & $1021^{\mathrm{a}}$ & & & 1046 & 1055 & 1053 & & & & & & & & \\
\hline 875 & 865 & 873 & & 870 & & 873 & 879 & 852 & 863 & & 859 & & & 866 \\
\hline $\begin{array}{l}455 \\
413\end{array}$ & & & & & & & & & & 037 & & 047 & & $\begin{array}{l}438 \\
388\end{array}$ \\
\hline & & & & & & & $376^{a}$ & & 381 & & & 382 & 238 & \\
\hline 224 & 220 & 224 & 216 & 220 & 219 & 224 & & 225 & & 226 & 223 & & & $222^{\mathrm{a}}$ \\
\hline & $1 / 2$ & & 102 & & $99^{\mathrm{a}}$ & $102^{\mathrm{a}}$ & & $\begin{array}{l}98 \\
75\end{array}$ & $112^{\mathrm{a}}$ & $110^{\mathrm{a}}$ & 74 & 117 & $98^{\mathrm{a}}$ & \\
\hline 70 & & 68 & & 70 & 70 & 70 & 70 & & 72 & 73 & & & & 71 \\
\hline
\end{tabular}

${ }^{\mathrm{a}}$ refers to weak bands 
Table 3 Molecular weight of bands following HpaII digestion of the apxIVA amplification product of the reference strains of A. pleuropneumoniae.

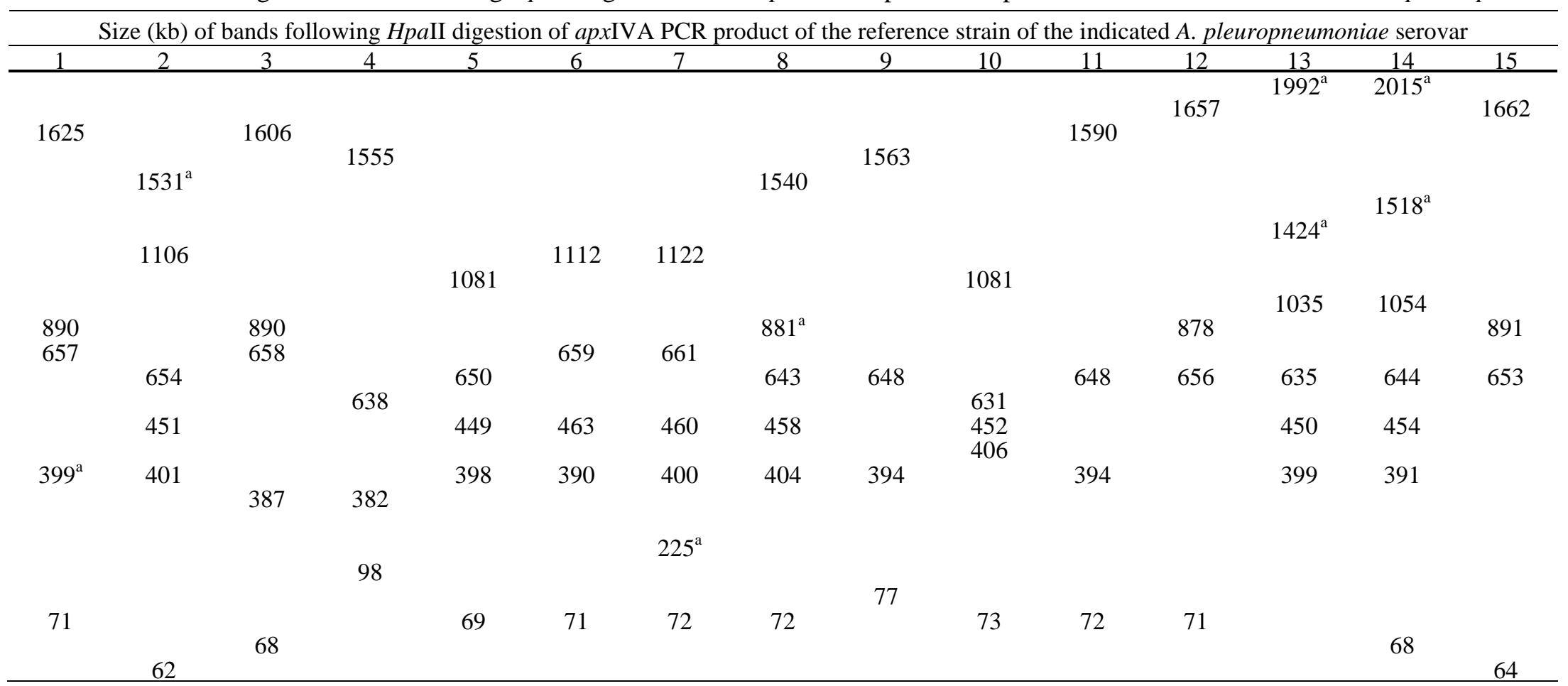

${ }^{a}$ Faint band 
Table 4 Summary of outcome of serovar assignment by PCR-REA of the apxIVA gene using either $C f o I$ or HpaII.

\begin{tabular}{ccccc}
\hline \multirow{2}{*}{$\begin{array}{c}\text { GD /IHA } \\
\text { serovar }\end{array}$} & $\begin{array}{c}\text { Total Number } \\
\text { of Isolates }\end{array}$ & \multicolumn{2}{c}{ Results of serovar assignment by PCR-REA method } \\
\cline { 3 - 5 } & & $\begin{array}{c}\text { Matches with } \\
\text { Serotyping }\end{array}$ & $\begin{array}{c}\text { Near Match with } \\
\text { Serotyping }\end{array}$ & $\begin{array}{c}\text { Mismatch with } \\
\text { Serotyping }^{\mathrm{b}}\end{array}$ \\
\hline 1 & 8 & 0 & 2 & 6 \\
2 & 2 & 2 & 0 & 0 \\
3 & 4 & 0 & 1 & 3 \\
5 & 5 & 3 & 0 & 2 \\
7 & 4 & 2 & 1 & 1 \\
8 & 7 & 7 & 0 & 0 \\
9 & 1 & 0 & 0 & 1 \\
12 & 4 & 0 & 3 & 1 \\
15 & 8 & 7 & 0 & 15 \\
Total & 43 & 21 & 7 & 1 \\
\hline
\end{tabular}

${ }^{a}$ Near match defined as meaning that the PCR-REA method suggested more than one serovar with one of those serovars matching the serovar identified by GD/IHA testing

${ }^{\mathrm{b}}$ Mismatch defined as meaning that the PCR-REA method suggested a serovar or serovars that did not match with the GD/IHA testing or resulted in a pattern not present in the serovar reference strains. 